\title{
Metagenomic and volatile profiles of ripened cheese obtained from dairy ewes fed a dietary hemp seed supplementation
}

\author{
A. lanni, ${ }^{1} \odot$ M. Di Domenico, ${ }^{2} \odot$ F. Bennato, ${ }^{1} \odot$ A. Peserico, ${ }^{2}$ C. Martino, ${ }^{2}$ A. Rinaldi, ${ }^{2}$ L. Candeloro, ${ }^{2}$ \\ L. Grotta, ${ }^{1}$ C. Cammà, ${ }^{2}$ F. Pomilio, ${ }^{2}$ and G. Martino ${ }^{1 *}$ (i) \\ ${ }^{1}$ Faculty of BioScience and Technology for Food, Agriculture and Environment, University of Teramo, Via Renato Balzarini 1, 64100 Teramo, Italy \\ ${ }^{2}$ Istituto Zooprofilattico Sperimentale dell'Abruzzo e del Molise G. Caporale, Via Campo Boario, 64100 Teramo, Italy
}

\begin{abstract}
Chemical and organoleptic properties of dairy products largely depend on the action of microorganisms that tend to be selected in cheese during ripening in response to the availability of specific substrates. The aim of this work was to evaluate the effects of a diet enriched with hemp seeds on the microbiota composition of fresh and ripened cheese produced from milk of lactating ewes. Thirty-two half-bred ewes were involved in the study, in which half (control group) received a standard diet, and the other half (experimental group) took a diet enriched with 5\% hemp seeds (on a DM basis) for $35 \mathrm{~d}$. The dietary supplementation significantly increased the lactose in milk, but no variations in total fat, proteins, caseins, and urea were observed. Likewise, no changes in total fat, proteins, or ash were detected in the derived cheeses. The metagenomic approach was used to characterize the microbiota of raw milk and cheese. The phyla Proteobacteria and Firmicutes were in equally high abundance in both control and experimental raw milk samples, whereas Bacteroidetes was less abundant. The scenario changed when considering the dairy products. In all cheese samples, Firmicutes was clearly predominant, with Streptococcaceae being the most abundant family in the experimental group. The reduction of taxa observed during ripening was in accordance with the increment (relative abundance) of the starter culture Lactococcus lactis and Streptococcus thermophilus, which together dominate the microbial community. The analysis of the volatile profile in ripened cheeses led to the identification of 3 major classes of compounds: free fatty acids, ketones, and aldehydes, which indicate a prevalence of lipolysis compared with the other biochemical mechanisms that characterize the cheese ripening.
\end{abstract}

Received November 24, 2019.

Accepted February 29, 2020.

*Corresponding author: gmartino@unite.it
Key words: hemp seed, dairy ewe, volatile compound, metagenomic

\section{INTRODUCTION}

Many of the chemical and organoleptic properties of cheeses, especially in ripened products, depend on the biochemical processes of the microorganisms embedded in the cheese matrix. This applies to both dairy products produced from raw milk and products produced after the milk pasteurization process and the addition of starter microorganisms. The bacteria found in the final ripened dairy product are more able than other microorganisms to use the available substrates, adapting their metabolism to the physicochemical variations of the cheese environment and, as a consequence, displacing other competitors during the ripening process (Escobar-Zepeda et al., 2016; Schirone et al., 2018). The role of each organism in the dairy product matrix is not yet fully understood in terms of the production of compounds related to flavor and aroma. However, regarding food safety, at the end of the ripening time, there is generally a decrease of gram-negative population, including pathogen and coliform bacteria, due to physicochemical changes that lead to the production of antimicrobial compounds (Beresford and Williams, 2004).

Over time, interest has grown in the development of feeding strategies to improve the chemical-nutritional properties of milk and dairy products, assuming that nutrition can influence milk composition in ruminants (Chaves Lopez et al., 2016; Bennato et al., 2019; Ianni et al., 2019a). These changes can certainly influence the microbiological profile of dairy products during the ripening period. For that reason, the aim of this work was to test the effects of a diet enriched with hemp seed on the chemical characteristics of milk and cheese from dairy ewes, and to correlate these variations to the microbiota characterized in the cheese environment during the ripening period. In a recent work, the inclusion of hemp seed (HS) in the diet of dairy ewes was 
effective in improving the milk fatty acids (FA) profile by decreasing the concentration of SFA and the $n-6 / n-3$ ratio, and increasing the amount of trans-vaccenic acid (C18:1,trans-11) and CLA (Mierliță, 2016). Conjugated linoleic acid, in particular rumenic acid (cis-9,trans-11 CLA), has been shown to perform an important antioxidant activity that protects bovine mammary epithelial cells from lipoperoxidation and mitigates the level of the reactive oxygen species, leading to an improvement of mammary gland functionality (Basiricò et al., 2017).

Culture-based microbiology techniques have been used to understand the microbial component of cheese. However, it has become increasingly clear that this approach often fails to detect "difficult-to-culture" or sub-dominant microorganisms. The advent of highthroughput sequencing technologies and the use of metagenomics have allowed comprehensive profiling of microbial diversity in milk and dairy products, without the need for culturing (Jung et al., 2011; Nalbantoglu et al., 2014; Falardeau et al., 2019).

Recently, this strategy has been used to investigate the metabolic potential in samples of naturally aged cheeses (Wolfe et al., 2014) and to monitor the variations of microbial abundance in a surface-ripened cheese through time (Dugat-Bony et al., 2015). This study therefore aims to evaluate the microbiota composition of fresh and ripened cheese obtained from lactating ewes fed with a hemp seed supplementation.

\section{MATERIALS AND METHODS}

\section{Experimental Design, Diets, and Cheese Manufacturing Protocol}

The experimental plan was performed according to Directive 2010/63/EU of the European Parliament (European Union, 2010) and Directive 86/609/EEC (European Economic Community, 1986), which deal with the protection of animals used for scientific purposes.

Thirty-two half-bred ewes, homogeneous for age (43 $\pm 3 \mathrm{mo}$ ), days in milking ( $74 \pm 4 \mathrm{~d}$ ), and weight (59 $\pm 4 \mathrm{~kg}$ ), were randomly divided into 2 groups of 16 : a control group (CG), whose diet received no supplementation, and an experimental group (EG), whose diet was supplemented with $5 \%$ HS on a DM basis.

The trial was conducted for $35 \mathrm{~d}$. At the end of the treatment, bulk milk was collected from the EG and manipulated in the same way during cheesemaking, according to the manufacturing protocol reported below. Bulk milk was heated at $70 \pm 1^{\circ} \mathrm{C}$ for $15 \mathrm{~s}$, cooled at $40^{\circ} \mathrm{C}$ and inoculated with a starter culture (Lactococcus lactis, Streptococcus thermophilus) produced by MOFIN ALCE Group (Novara, Italy). Then, the milk was coagulated by adding kid rennet paste, and the curd was subsequently cut into pieces similar in size to corn kernels, heated to $42 \pm 1^{\circ} \mathrm{C}$, and manually pressed. The resulting cheeses, of about $1 \mathrm{~kg}$ each, were held at $10^{\circ} \mathrm{C}$ until the next day, when they were salted in aqueous solution containing $18 \% \mathrm{NaCl}$ (sodium chloride). Ripening was conducted at $12 \pm 1^{\circ} \mathrm{C}$. To evaluate changes in the chemical composition and quality attributes due to ripening, sampling and analysis of pecorino cheese were carried out at $1 \mathrm{~d}$ (T1), $30 \mathrm{~d}$ (T30) and $120 \mathrm{~d}$ (T120) after the cheesemaking. Samples, collected in triplicate from 3 different cheesemaking sessions, were either analyzed immediately or vacuum-packed and frozen at $-20^{\circ} \mathrm{C}$ until analysis.

\section{Chemical Analysis of Milk and Pecorino Cheese}

The MilkoScan FT 6000 (Foss Integrator IMT; Foss, Hillerød, Denmark) was used to determine the chemical composition of milk (fat, protein, casein, lactose, and urea).

For the cheese, AOAC International (2000) methods were used to determine the moisture content (method 933.05), total protein (method 920.123), and ash (method 935.42). For the evaluation of total lipids, cheese samples were subjected to acid hydrolysis. About $1 \mathrm{~g}$ of each sample was heated at $80^{\circ} \mathrm{C}$ for $30 \mathrm{~min}$ in the presence of $20 \mathrm{~mL}$ of ethanol and $500 \mu \mathrm{L}$ of $\mathrm{HCl}$ (hydrochloric acid) $3.0 \mathrm{~N}$. After cooling at room temperature, $2 \mathrm{~mL}$ of distilled water, $8 \mathrm{~mL}$ of petrolatum ether, and $1 \mathrm{~g}$ of $\mathrm{NaCl}$ were added to each sample. Samples were shaken, sonicated, and centrifuged at 2,000 $\times g$ at room temperature for $5 \mathrm{~min}$ to facilitate the separation of the solution in 2 phases. The supernatants containing non-saponifiable lipids were recovered in calibrated round-bottom flasks, and the solvent of each sample was evaporated to dryness with a Strike-Rotating Evaporator (Steroglass S.r.l., Perugia, Italy) at $38^{\circ} \mathrm{C}$. Then, all the flasks were incubated in a stove for $1 \mathrm{~h}$ at $50^{\circ} \mathrm{C}$. After cooling to room temperature, they were weighed to calculate the total lipids percentage.

\section{DNA Extraction from the Cheese}

The total DNA was extracted from milk and cheese samples in triplicate. Samples were preprocessed to remove fats and concentrate bacterial cells. A total of 2 $\mathrm{g}$ was sampled from different parts of the cheese block and pooled to obtain a representative sample, which was placed in a stomacher bag with $20 \mathrm{~mL}$ of PBS and homogenized for $5 \mathrm{~min}$. The milk samples were shaken vigorously for $10 \mathrm{~s}$ before centrifugation. The cheese solution and milk were clarified by centrifugation at $2,500 \times g$ for $3 \mathrm{~min}$ at room temperature. The fatty 
layer was removed, and supernatant was collected and centrifuged at room temperature for $5 \mathrm{~min}$ at 14,000 $\times g$. Then, the supernatant was discarded and the pellets were resuspended in $400 \mu \mathrm{L}$ of PBS. Finally, the resuspended pellets were transferred to the cartridges provided by the Maxwell 16 Tissue DNA Purification Kit (Promega, Madison, WI) for automatic DNA extraction on the Maxwell 16 Instrument (Promega). The DNA was then quantified by Qubit dsDNA HS Assay (Thermo Fisher Scientific, Waltham, MA) and used for metagenomic analysis by MinION.

\section{Metagenomic Shotgun Sequencing with MinION}

Libraries were prepared with the low-input genomic DNA by PCR Barcoding (SQK-PBK004, Oxford Nanopore Technologies, Oxford, UK), following manufacturer's guidelines. The DNA ends were end-repaired and dA-tailed using the NEBNext End Repair/dATailing module (New England Biolabs, Hitchin, UK). The PCR adapters were ligated onto the prepared ends using the Blunt/TA Ligase Master Mix (New England Biolabs), and a unique barcode was added by PCR. The amplification program consisted of a 3-min step at $95^{\circ} \mathrm{C}$, followed by 14 cycles with the following conditions: $95^{\circ} \mathrm{C}$ for $15 \mathrm{~s}, 56^{\circ} \mathrm{C}$ for $15 \mathrm{~s}$, and $65^{\circ} \mathrm{C}$ for 6 min. The program ended with 1 step at $65^{\circ} \mathrm{C}$ for 6 min. Sequencing adapters were added prior to library loading on the Flow cell MIN106, R9 version (Oxford Nanopore Technologies). All purification steps were carried out using AMPure XP beads (Agencourt, Beckman Coulter, Brea, CA) according the SQK-PBK004 sequencing protocol. For sequencing, the NC_48hr_sequencing_FLO-MIN106_SQK-PBK004 program was run on MinKNOW Software v.19.05.0. The 24 samples were sequenced in 2 consecutive runs.

\section{Bioinformatic Analysis}

MinION data was analyzed as previously reported with some modification (Beato et al., 2018; Peserico et al., 2019). Briefly, Fast5 raw MinION files were processed locally using the Guppy software (version 3.1.5; https://community.nanoporetech.com/downloads) for base calling. Barcoding was performed by Porechop software (version 0.2.3; https://github.com/rrwick/ Porechop). These steps obtained FASTQ files output with a quality cut-off fixed as > Q7. Quality check analysis of raw reads was performed by using Nanoplot (version 1.17.2; De Coster et al., 2018). Taxonomic profiling of metagenomic sequences was done by Centrifuge tool (version 1.0.3; Kim et al., 2016). Reads assigned to viruses and phages were removed.

\section{$\alpha$ - and $\beta$-Diversity}

$\alpha$-Diversity defines the variety and abundance of species in a microbial community. The complexity is generally described by different indexes, which weight the 2 components, richness and evenness, in different manners. The commonly used $\alpha$-diversity indexes include species observed, Shannon index, and Simpson index. Species observed is equal to richness, and therefore, provides no weight to the evenness component; Shannon index weights richness and evenness equally; and Simpson index provides more weight to evenness. $\beta$-Diversity indexes are calculated for each pair of groups and represent either a similarity or difference between the 2 groups.

The taxonomic classification generated by centrifuge was used to measure $\alpha$ - and $\beta$-diversity in $R$ package (software for statistical computing and graphics) by both betapart and Phyloseq (Baselga and Orme, 2012; McMurdie and Holmes, 2013). Using betapart, $\alpha$-diversity was measured by richness (total operational taxonomic units, OTU) and evenness (Shannon Index), whereas $\beta$-diversity was calculated by Jaccard dissimilarity $\left(\beta_{\mathrm{jac}}\right)$. Different indexes of $\alpha$-diversity $(\mathrm{Ob}-$ served OTU, Chao1, ACE, Shannon, Simpson, inverse Simpson, and Fisher) were also calculated by Phyloseq, and for $\beta$-diversity a pairwise permutational multivariate ANOVA (PERMANOVA) test was conducted using pairwiseAdonis (Martinez-Arbizu, 2019).

\section{Volatile Compounds Analysis}

The extraction and analysis of volatile compounds (VOC) from cheese samples were performed according to the procedure reported by Ianni et al. (2019c), with slight modifications. Five grams of grated cheese was mixed with $10 \mathrm{~mL}$ of saturated $\mathrm{NaCl}$ solution $(360 \mathrm{~g} / \mathrm{L})$, and the mixture was homogenized with Ultra Turrax T-25 high speed homogenizer (IKA, Staufen, Germany). Then, $10 \mu \mathrm{L}$ of internal standard solution (4-methyl2-heptanone; $10 \mathrm{mg} / \mathrm{kg}$ in ethanol) was added, and the vials were sealed with a polytetrafluoroethylene-silicone septum (Supelco, Bellefonte, PA) and stirred at $60^{\circ} \mathrm{C}$. The VOC were extracted from the headspace with a divinylbenzene-Carboxen-polydimethylsiloxane solidphase microextraction (SPME) fiber (length: $1 \mathrm{~cm}$; film thickness: $50 / 30 \mu \mathrm{m}$; Supelco) with an exposition time of $50 \mathrm{~min}$. The extracted VOC were analyzed by a gas chromatograph (Clarus 580; Perkin Elmer, Waltham, MA) coupled with a mass spectrometer (SQ8S; Perkin Elmer). The gas chromatograph was equipped with an Elite-5MS column (length $\times$ internal diameter: 30 $\times 0.25 \mathrm{~mm}$; film thickness: $0.25 \mu \mathrm{m}$; Perkin Elmer). 
The VOC were thermally desorbed into the gas chromatograph injector in a splitless mode for $1 \mathrm{~min}$ at $250^{\circ} \mathrm{C}$. The oven temperature was held at $50^{\circ} \mathrm{C}$ for 1 min, increased at a rate of $3^{\circ} \mathrm{C}$ per min up to $200^{\circ} \mathrm{C}$ and held for $1 \mathrm{~min}$, and then increased from $200^{\circ} \mathrm{C}$ to $250^{\circ} \mathrm{C}$ at $15^{\circ} \mathrm{C}$ per min and held for $20 \mathrm{~min}$. Helium was used as a carrier gas at a flow rate of $1 \mathrm{~mL} / \mathrm{min}$. The mass spectrometer operated in electron impact ionization mode at $70 \mathrm{eV}$, and data were collected in full-scan mode, with a scan time of $0.2 \mathrm{~s}$ over a mass range from 35 to 350 . Source and interface temperature were held at $250^{\circ} \mathrm{C}$. The VOC were identified by comparison with mass spectra of a library database (NIST Mass Spectral library, Search Program version 2.0, National Institute of Standards and Technology, Gaithersburg, MD) and by comparing the eluting order with Kovats indexes.

\section{Statistical Analysis}

Experiments were conducted at least in triplicate. The data were analyzed statistically using SigmaPlot 12.0 software (Systat Software Inc., San Jose, CA) for Windows operating system. The data were tabulated as mean \pm standard deviation. One-way ANOVA was performed. Significantly different groups were ranked using the post hoc comparison tests (Tukey test) at 95\% $(P<0.05)$ confidence level.

\section{RESULTS AND DISCUSSION}

\section{Chemical Composition of Milk and Cheese}

The enrichment of dairy ewes' diet with HS did not induce in milk quantitative changes in total fat, proteins, caseins, and urea (Table 1), nor were any changes found in $\mathrm{pH}$; however, the diet supplemented with HS significantly increased the lactose concentration (4.69 $\pm 0.41 \%$ vs. $5.84 \pm 0.49 \%$ in $\mathrm{CG}$ and EG respectively; $P<0.01)$.

Table 1. Chemical composition of milk obtained from the control group (CG; no supplementation) and the experimental group (EG; fed hemp seeds at $5 \%$ of diet DM)

\begin{tabular}{lrrr}
\hline & \multicolumn{2}{c}{ Diet $^{1}$} \\
\cline { 2 - 3 } Item & \multicolumn{1}{c}{ CG } & \multicolumn{1}{c}{ EG } & \multirow{2}{*}{$P$-value } \\
\hline Fat, \% & $6.40 \pm 0.72$ & $5.96 \pm 0.62$ & NS \\
Protein, \% & $5.25 \pm 0.49$ & $5.17 \pm 0.31$ & NS \\
Casein, \% & $4.08 \pm 0.39$ & $4.00 \pm 0.23$ & NS \\
Lactose, \% & $4.69 \pm 0.41$ & $5.84 \pm 0.49$ & $* *$ \\
Urea, mg/100 mL & $21.07 \pm 1.34$ & $20.93 \pm 1.08$ & NS \\
pH & $6.47 \pm 0.05$ & $6.51 \pm 0.05$ & NS \\
\hline
\end{tabular}

${ }^{1}$ Data are reported as mean \pm standard deviation.

$* * P<0.01$.
As in milk, the dietary supplementation did not influence the chemical composition of cheese in terms of total fat, proteins, and ash. As expected, the only significant variation was in DM, which significantly increased during ripening, but there were no differences related to diet.

\section{Microbiota Characterization of Cheese During Ripening}

A total of 117,100 reads were obtained by MinION shotgun sequencing in 2 consecutive runs. There were 77,900 unclassified reads, whereas 39,100 were classified as microbial reads. Of those, 29,900 were bacterial and 8,800 were viral, which were not considered for microbiota characterization because they were not involved in metabolic pathways.

The phyla Proteobacteria and Firmicutes were in equally high abundance within milk samples (CG and EG), whereas Bacteroidetes was less abundant. Bacillaceae, Flavobacteriaceae, and Clostridiaceae, in descending order, were the dominant families. Details of family abundance are illustrated in Figure 1. The scenario changed when considering the use of the starter culture in cheese products. Indeed, the dominant phylum in all fresh cheese samples (T1), independent of diet, was clearly Firmicutes, with the most abundant family being Streptococcaceae. Bacillaceae, Flavobacteriaceae, and Clostridiaceae remained in the same order of abundance, but were less represented. The starter growth in ripened-cheese samples (T30, T120) determined the absolute prevalence of the family Streptococcaceae.

The $\alpha$-diversity was the highest overall in milk and cheese samples (CG and EG) at T1, and lowest in the cheese samples (CG and EG) at T30 and T120 (Table 2, Figure 2), according to all indexes used. The reduction of taxa during ripening was in accordance with the increment (relative abundance) of the starter culture $L$. lactis and $S$. thermophilus, which together dominated the microbial community (97\% in CG and $68 \%$ in EG). Details of the starter culture in ripened cheeses are reported in Figure 3. The EG cheese samples (T30 and T120) showed higher OTU and Shannon Index values than the CG cheese samples (Table 3).

Dietary HS supplementation did not influence $\beta$-diversity in milk $(P>0.05)$ or cheeses from T1 to T120 $(P>0.05)$, despite some extant differences between the CG and EG in cheese at T30 and T120 (Figure 4).

\section{Volatile Profile of Pecorino Cheese}

The analysis of VOC of T1, T30, and T120 cheese samples from the CG and EG identified several chemi- 


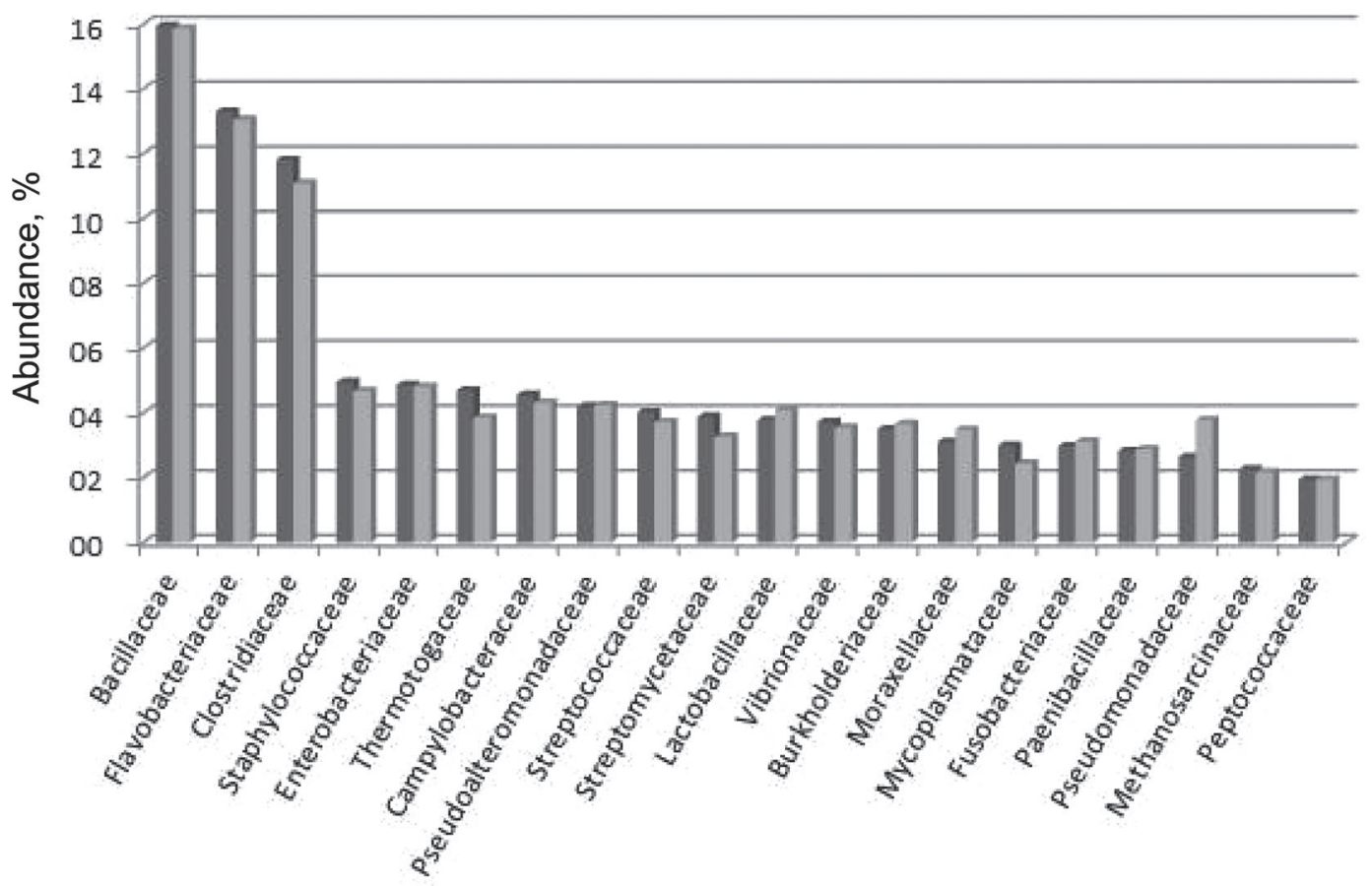

Figure 1. Most represented families of bacteria and relative abundance observed in milk samples obtained from the control group (CG, no supplementation; dark gray bars) and the experimental group (EG, fed hemp seeds at $5 \%$ of diet DM; light gray bars).

cal families. The most abundant compounds were free fatty acids (FFA), followed by ketones and aldehydes. The greater concentration of FFA in all the analyzed samples indicated the prevalence of lipolysis relative to proteolysis.

The results at $\mathrm{T} 1$ showed a marked difference in FFA between cheese samples $(48.20 \%$ vs. $89.96 \%$ for CG and EG, respectively; $P<0.01$ ). This difference was reduced during ripening until FFA reached comparable values at T120 (83.07\% vs. $95.78 \%$ for CG and EG, respectively; $P>0.05)$. As shown in Figure 5, the lack of a significant difference between the groups in total FFA at T120 was due to an increase in FFA concentration in the CG cheese samples. In fact, the sum of FFA in the EG samples did not undergo significant changes during ripening, remaining around $90 \%$ of the total detected VOC. Table 3 shows the detailed FFA composition in the various cheese samples. Concentrations of butanoic, hexanoic, and octanoic acids increased in the CG samples during ripening, whereas that of decanoic acid decreased significantly $(P<0.01$ for all compounds). Concentrations of butanoic and hexanoic acids increased $(P<0.05)$ during ripening in the EG samples, although the variation was not as marked as in the CG samples; no significant changes were noted for the other compounds.

Higher concentrations of ketones (Figure 5) were detected in the CG samples in all ripening times. In both the CG and EG samples, the greatest concentration of ketones was reached at T30; after that, there was an overall decrease in these compounds, which were lower at $\mathrm{T} 120$ than at $\mathrm{T} 1$.

Dietary HS supplementation negatively influenced the synthesis of aldehydes in cheeses. In fact, as shown in Figure 5, the concentration of these compounds was very low in the EG samples, with no notable changes

Table 2. $\alpha$-Diversity measured as the richness (operational taxonomic units, OTU) and evenness (Shannon) calculated by betapart (Baselga and Orme, 2012) ${ }^{1}$

\begin{tabular}{|c|c|c|c|c|c|c|c|c|}
\hline Measure & \multicolumn{2}{|c|}{ Milk } & \multicolumn{2}{|c|}{ Cheese T1 } & \multicolumn{2}{|c|}{ Cheese T30 } & \multicolumn{2}{|c|}{ Cheese T120 } \\
\hline Observed OTU & 2,963 & 2,768 & 2,722 & 2,705 & 185 & 295 & 133 & 289 \\
\hline
\end{tabular}


during ripening. The results for the CG samples were quite different. The concentration of aldehydes was $12.23 \%$ at $\mathrm{T} 1$ and drastically reduced during ripening, reaching $6.64 \%$ at T30 $(P<0.01)$ and $2.10 \%$ at T120 $(P<0.01)$.

Enriching the diet of ewes with HS led to interesting variations in dairy products at the end of the experimental period. Analysis of the milk used for cheesemaking showed a significantly higher concentration of lactose in the EG, affecting the metabolism of bacteria present in the cheese matrix, which are responsible for the biochemical processes that affect organoleptic properties during ripening. The variation in lactose concentration, as a function of the diet fed to the animals, has been reported in other studies. Luo et al. (2018) reported an increase in concentrations of fat and lactose in colostrum and milk obtained from dairy ewes in early lactation that were supplemented with soy isoflavones. In the same study, the authors demonstrated a role for isoflavones in the increased concentration of serum prolactin, which improves mammary gland function. Similar studies in cows indicated that daidzein and genistein, other types of isoflavones, increased the proliferation and development of mammary epithelial cells, milk fat and protein, and milk yield (Liu et al., 2012; Liu et al., 2013). Isoflavones are widely represented in HS and are fundamental components of the total polyphenol content, together with flavanones, flavonols, and flavanols (Smeriglio et al., 2016). It is therefore plausible that in our study, these compounds, having bioactive properties, improved mammary gland functionality and influenced the nutritional properties of milk. Recently, it has been shown that the lactose concentration in milk can increase as a result of feeding a diet rich in PUFA to dairy ewes (Hamer et al., 2018). In this regard, one of the best-characterized properties of HS is its FA composition, which comprises essential constituents belonging to n-3 and n- 6 classes, which are necessary for many physiological processes, including the maintenance of cell-membrane structure (Crescente et al., 2018). In this study, both milk and derived cheese samples showed a significant increase in the total amount of PUFA (Supplemental Table S1; https://doi.org/10.3168/jds.2019-17954), mainly due to the increase in concentrations of linoleic acid, linolenic acid, and CLA. This effect at the cellular level could

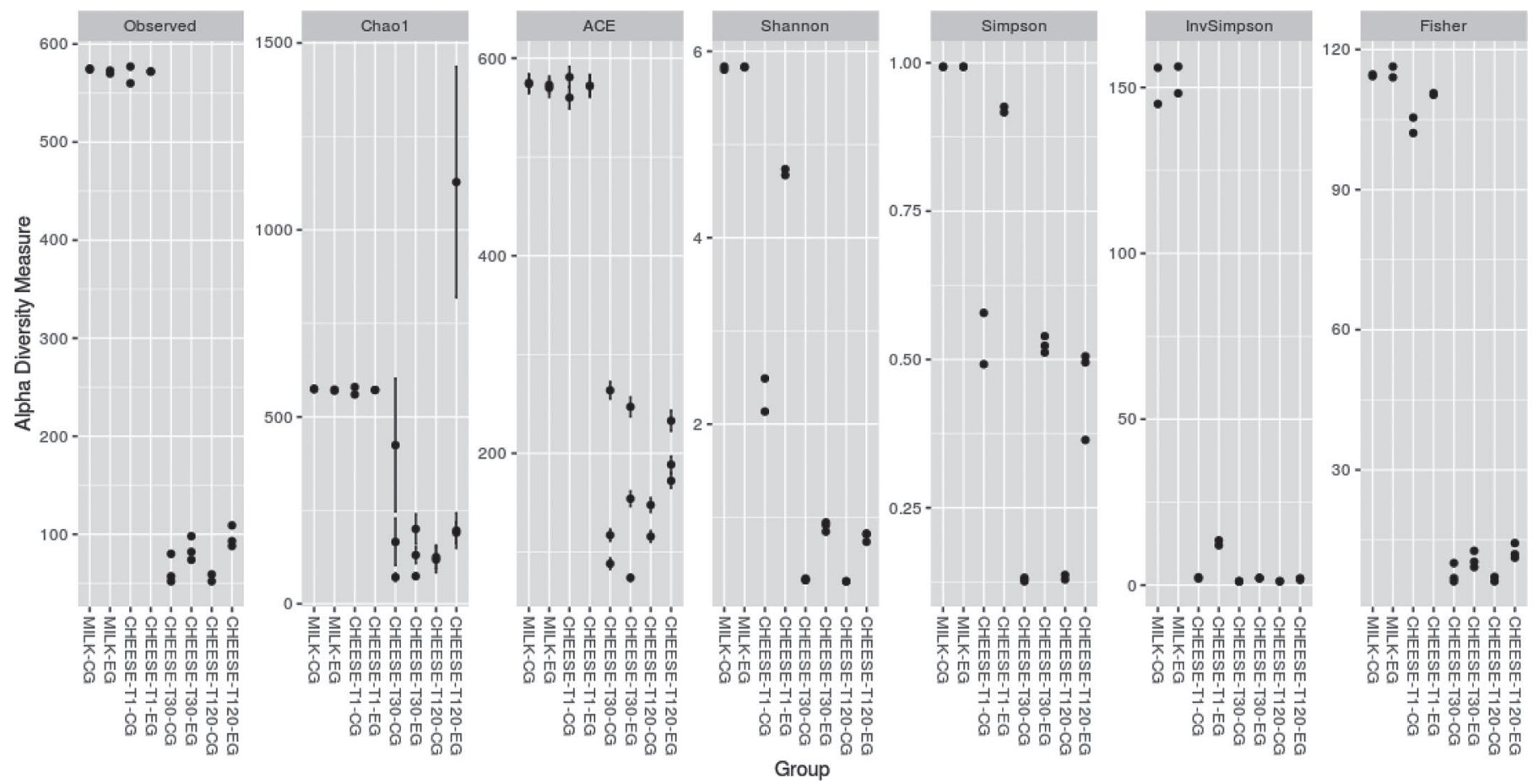

Figure 2. $\alpha$-Diversity measured by Observed OTU (operational taxonomic units), Chao1, ACE (abundance-based coverage estimator), Shannon, Simpson, inverse Simpson, and Fisher indexes, calculated in milk and cheese samples of the control group (CG, no supplementation) and the experimental group (EG, fed hemp seeds at 5\% of diet DM) after $1 \mathrm{~d}$ (T1), $30 \mathrm{~d}$ (T30) and $120 \mathrm{~d}$ (T120) of ripening by Phyloseq (McMurdie and Holmes, 2013). Milk and cheeses were pasteurized. The starter culture (Streptococcus thermophilus and Lactococcus lactis) was added for cheese production for both CG and EG. 


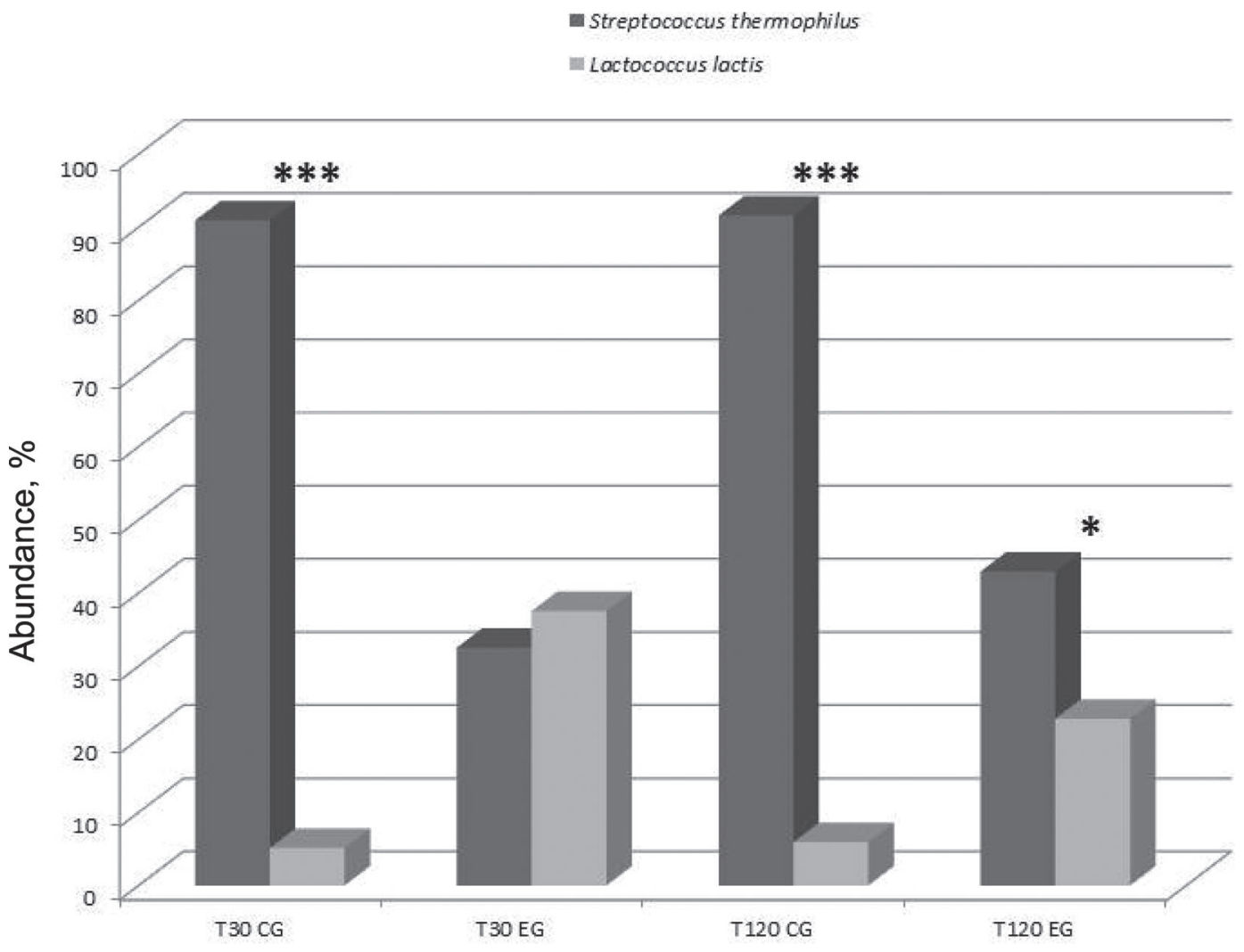

Figure 3. Relative abundance of the starter culture (Streptococcus thermophilus and Lactococcus lactis) after $30 \mathrm{~d}$ (T30) and $120 \mathrm{~d}(\mathrm{~T} 120)$ of ripening in both control group (CG, no supplementation) and experimental group (EG, fed hemp seeds at $5 \%$ of diet DM). ${ }^{*} P<0.05 ; * * * P$ $<0.001$.

partly support the hypothesis of an improvement in the functionality of the mammary gland, with a consequent increase in lactose concentration in milk.

In light of this finding, we expected a reduction in the $\mathrm{pH}$ value; however, this did not happen, and the reasons may be manifold. As previously reported by Salaün et al. (2005), several compositional factors can influence the buffering capacity of dairy products, in particular, the concentration of small constituents (inorganic phosphate, citrate, and organic acids) and milk proteins, such as caseins and whey proteins.

The cheesemaking process for pecorino cheese includes a thermal treatment in the first step and the addition of starter cultures. As expected, this manufacturing protocol greatly reduced the heterogeneity of the microbiota. However, the metagenomic approach was very useful to describe $\alpha$ - and $\beta$-diversity and the relative abundance of the microbial species from milk to the cheese during different steps of the ripening period for both CG and EG. The microbial composition of milk is generally influenced by different factors. The microorganisms present in the teat canal and on the teat surface, in the air circulating inside the farm, in the feed, and other environmental factors (i.e., water supply, general hygiene), all contribute to milk microbial diversity (Quigley et al., 2013; Montel et al., 2014). In this study, no significant differences were observed between CG and EG in milk or cheese at T1 or along the ripening process. The microbial diversity was thus not influenced by the dietary hemp seed supplementation. On the other hand, differences in the growth rate observed in the starter cultures $S$. thermophilus and L. lactis emerged between the CG and EG and suggest that substrate changes (lactose) modified the relative abundance in ripened cheese (T30 and T120). In particular, the CG microbiota was dominated by $S$. thermophilus ( $\sim 90 \%$ ), with the remainder being $L$. lactis and a few other species. Conversely, the EG cheese showed a greater homogeneity between $S$. thermophilus and L. lactis and a greater number of species present in the cheese samples. Despite this difference, the richness of non-starter species between the 2 groups was small (Observed, Figure 2); the evenness (Simpson, Figure 2) defines a clearer scenario in which CG and EG are quite distant at T30 and T120. We could not determine whether the overgrowth of $S$. thermophilus led to the reduction of diversity in the $\mathrm{CG}$, or whether the greater diversity controlled the spread of $S$. thermophilus in the 


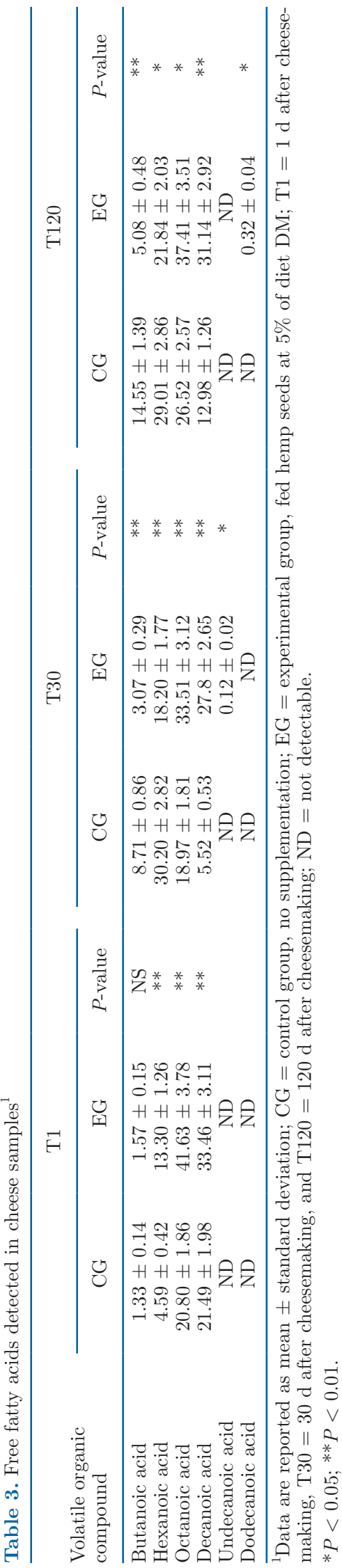

EG. Further microbiological trials are needed to understand these dynamics.

To date, the most evident difference between $L$. lactis and $S$. thermophilus is in the size of the genomes and thus, the number of genes (Pastink et al., 2009). The model of L. lactis contains 2,563 genes, compared with 1,889 genes in the $S$. thermophilus model. This would suggest more extensive metabolism pathways for $L$. lactis, but the total absolute numbers of reactions are similar: 522 for $S$. thermophilus and 598 for L. lactis. This parameter should demonstrate, at least in part, that the variations in the relative abundance of the 2 strains observed during ripening were determined by different capacities to adapt to the substrate present in the cheese matrix. In an attempt to correlate the observed increase in lactose in EG milk samples, with the metagenomic analysis, it should be noted that lactose is the main carbon and energy source for $L$. lactis and $S$. thermophilus, and it is metabolized by fermentation into L-lactate or D-lactate or a racemic mixture of both. This process represents an essential mechanism for the production of all cheese varieties; in fact, lactate has been reported to contribute to the flavor of acid-curd cheeses and ripened-cheese varieties (McSweeney and Sousa, 2000). Lactose enters the intracellular environment through the action of a lactose permease and hydrolysis to glucose and galactose by a $\beta$-galactosidase. With regard $S$. thermophilus, glucose is used by the glycolytic pathway, whereas galactose is not metabolized but is secreted in the medium by an antiporter, which allows cells to uptake additional lactose (Gunnewijk et al., 2001). Galactose accumulation in dairy products can lead to several problems, including toxic effects on consumers affected by galactosemia, a genetic disorder of galactose metabolism. Lactococcus lactis can metabolize galactose through a gene cluster that includes genes encoding a high-affinity galactose

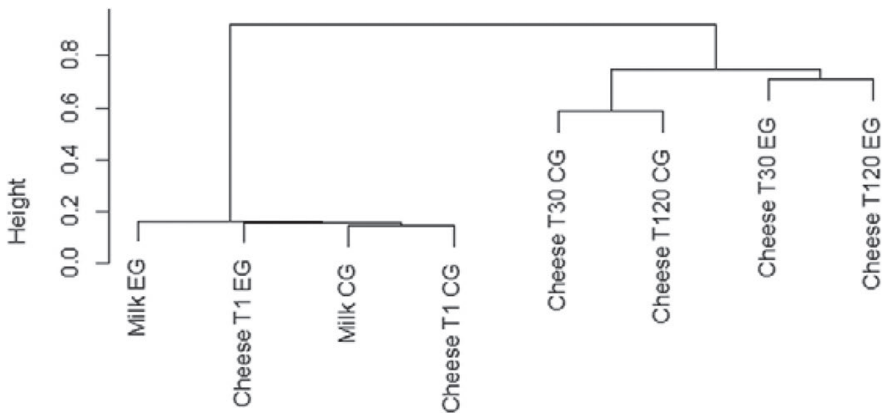

Figure 4. $\beta$-Diversity in milk and cheese samples after $1 \mathrm{~d}(\mathrm{~T} 1)$ and after ripening $30 \mathrm{~d}$ (T30) and $120 \mathrm{~d}$ (T120), obtained from both control group (CG, no supplementation) and experimental group (EG, fed hemp seeds at $5 \%$ of diet DM). $\beta$-Diversity was calculated by Jaccard dissimilarity $\left(\beta_{\text {jac }}\right)$. 
$\square \mathrm{CG}$

$\square \mathrm{EG}$
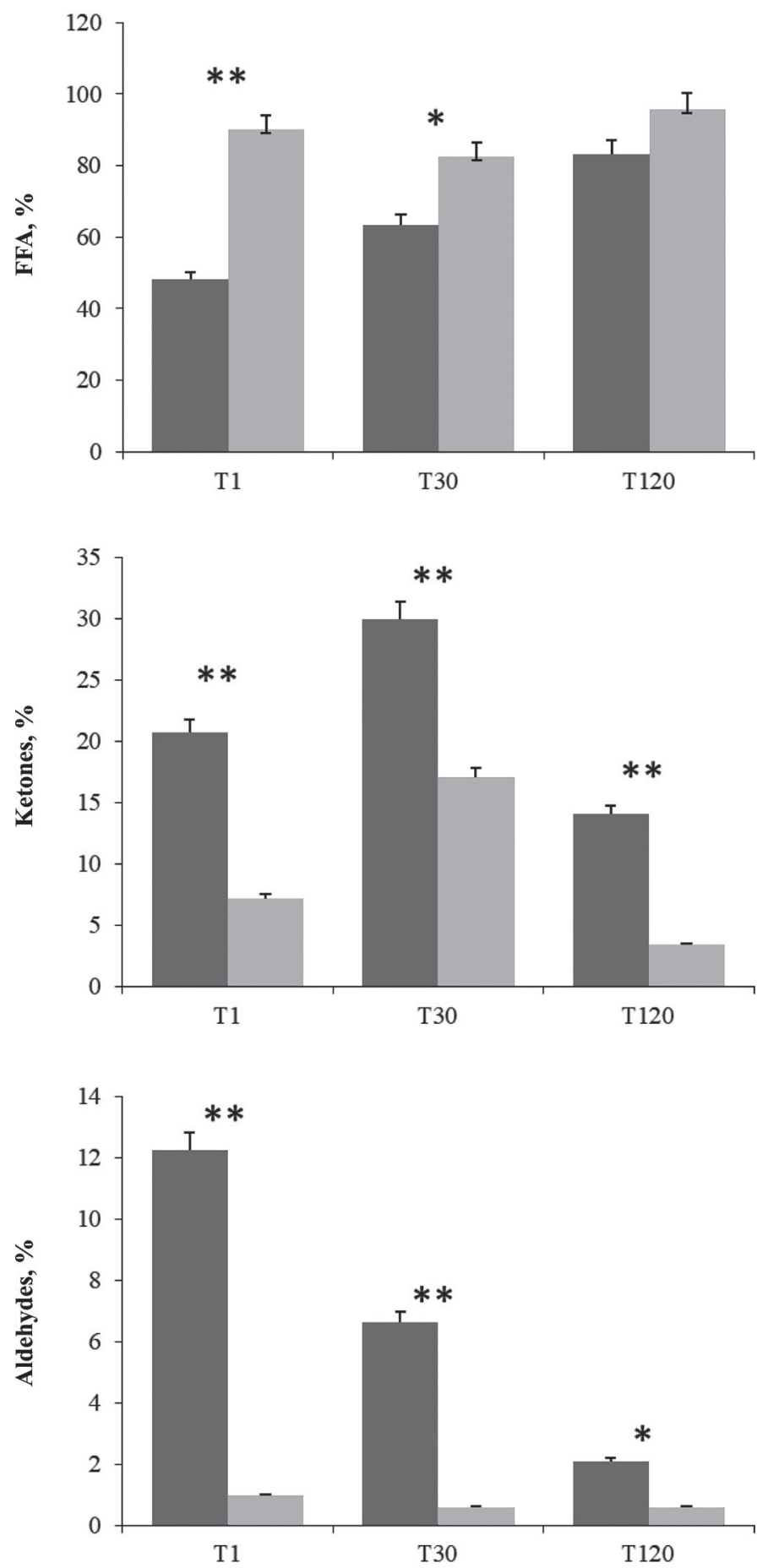

Figure 5. Most represented families of volatile compounds observed in cheese samples obtained from the control group (CG, no supplementation; dark columns) and the experimental group (EG, fed hemp seeds at $5 \%$ of diet DM; light columns) after $1 \mathrm{~d}$ (T1), $30 \mathrm{~d}$ (T30) and $120 \mathrm{~d}(\mathrm{~T} 120)$ of ripening. FFA $=$ free fatty acids. Data are reported as percentage values $\left({ }^{*} P<0.05\right.$; $\left.{ }^{*} P<0.01\right)$; error bars represent positive values of standard deviation. permease and enzymes involved in degradation of galactose (Grossiord et al., 1998). This would explain why $L$. lactis is better able to use lactose, which could explain, at least in part, the increase in L. lactis in the EG cheese samples during ripening.

Based on results from the metagenomics analysis, we evaluated the volatile profile of the cheeses to determine changes in VOC during ripening. The formation of VOC in dairy products is closely related to the substrates present in the matrix and the quantity and type of bacteria, which are selected during ripening in response to the substrate. The enrichment of ruminant diets with matrices rich in bioactive compounds can have direct effects on the volatile profile of dairy products at different ripening times (Ianni et al., 2019a,b; Bennato et al., 2020).

In this study, among the identified families of compounds, FFA were present in greater concentrations in all samples, consistent with prevalence of the lipolytic process compared with other catabolic mechanisms. The rennet paste used during cheesemaking, in addition to the proteolytic component consisting of chymosin (80-90\%) and pepsin (10-20\%), also has lipolytic potential, which contributes to the development of cheese aroma during ripening. The data reported in the literature on the activity of these enzymes are extremely variable, making it difficult to clarify their properties and specific contributions to cheese flavor. However, lipases commonly characterizing the rennet paste are mainly pregastric in origin and are highly specific for triglycerides containing short-chain FA (Collins et al., 2003). Indeed, the FA profile of both milk and cheese samples (Supplemental Table S1; https://doi.org/10 $.3168 /$ jds.2019-17954) showed that HS supplementation increased concentrations of SFA (from C4 to C10), confirming the prevalence of lipolytic mechanisms in EG samples, especially in the first part of the ripening. The FFA concentration in the EG samples begins at higher levels and remains almost unchanged at T1, T30, and T120, whereas in CG samples, the concentration of FFA increases progressively during ripening, reaching the levels of the EG samples only at T120.

This interesting behavior, in addition to the variations in individual FFA, may reflect the microbiological variations observed. In particular, concentrations of butanoic and hexanoic acids were increased in $\mathrm{CG}$ samples; these VOC, if identified in surface-ripened cheese types, are commonly reported to cause unpleasant aromatic notes defined as rancid, cheesy, and sweaty (Kilcawley, 2017). We detected few differences in concentrations of ketones and aldehydes among the samples analyzed during cheese ripening, and their contribution to the aromatic profile requires further and more specific evaluations. 


\section{CONCLUSIONS}

Dietary HS supplementation in ewes was effective in increasing the lactose content in milk. The metagenomic approach was very useful in evaluating the growth rate of the starter culture $S$. thermophilus and L. lactis in ewe milk cheese during ripening, leading to the characterization of microbial diversity in the EG, presumably due to the substrate change. Furthermore, we also noted interesting variations in cheese volatile profile during ripening, with potential effects on flavor.

\section{ACKNOWLEDGMENTS}

The authors have not stated any conflicts of interest.

\section{REFERENCES}

AOAC International. 2000. Official Methods of Analysis, 17th ed., Vol. II. AOAC International, Gaithersburg, MD.

Baselga, A., and C. D. L. Orme. 2012. Betapart: An R package for the study of beta diversity. Methods Ecol. Evol. 3:808-812. https://doi .org/10.1111/j.2041-210X.2012.00224.x.

Basiricò, L., P. Morera, D. Dipasquale, A. Tröscher, and U. Bernabucci. 2017. Comparison between conjugated linoleic acid and essential fatty acids in preventing oxidative stress in bovine mammary epithelial cells. J. Dairy Sci. 100:2299-2309. https://doi.org/ $10.3168 /$ jds.2016-11729.

Beato, M. S., M. Marcacci, E. Schiavon, L. Bertocchi, M. Di Domenico, A. Peserico, M. Mion, G. Zaccaria, L. Cavicchio, I. Mangone, E. Soranzo, C. Patavino, C. Cammà, and A. Lorusso. 2018. Identification and genetic characterization of bovine enterovirus by combination of two next generation sequencing platforms. J. Virol. Methods 260:21-25. https://doi.org/10.1016/j.jviromet.2018 .07 .002 .

Bennato, F., A. Ianni, D. Innosa, L. Grotta, A. D'Onofrio, and G. Martino. 2019. Chemical-nutritional characteristics and aromatic profile of milk and related dairy products obtained from goats fed with extruded linseed.Asian-Australas. J. Anim. Sci. https://doi .org/10.5713/ajas.18.0868. In press.

Bennato, F., A. Ianni, C. Martino, A. Di Luca, D. Innosa, A. M. Fusco, F. Pomilio, and G. Martino. 2020. Dietary supplementation of Saanen goats with dried licorice root modifies chemical and textural properties of dairy products. J. Dairy Sci. https://doi.org/10 $.3168 /$ jds.2019-16838. In press.

Beresford, T., and A. Williams. 2004. The microbiology of cheese ripening. Pages 287-318 in: Cheese: Chemistry, Physics and Microbiology, Vol. 2. Fox, P., P. McSweeney, T. Cogan, and T. Guinee, ed. Academic Press, Cambridge, MA.

Chaves Lopez, C., A. Serio, C. Rossi, G. Mazzarrino, S. Marchetti, F. Castellani, L. Grotta, F. P. Fiorentino, A. Paparella, and G. Martino. 2016. Effect of diet supplementation with Ascophyllum nodosum on cow milk composition and microbiota. J. Dairy Sci. 99:6285-6297. https://doi.org/10.3168/jds.2015-10837.

Collins, Y. F., P. L. H. McSweeney, and M. G. Wilkinson. 2003. Lipolysis and free fatty acid catabolism in cheese: A review of current knowledge. Int. Dairy J. 13:841-866. https://doi.org/10.1016/ S0958-6946(03)00109-2.

Crescente, G., S. Piccolella, A. Esposito, M. Scognamiglio, A. Fiorentino, and S. Pacifico. 2018. Chemical composition and nutraceutical properties of hempseed: An ancient food with actual functional value. Phytochem. Rev. 17:733-749. https://doi.org/10.1007/ s11101-018-9556-2.

De Coster, W., S. D'Hert, D. T. Schultz, M. Cruts, and C. Van Broeckhoven. 2018. NanoPack: Visualizing and processing longread sequencing data. Bioinformatics 34:2666-2669.
Dugat-Bony, E., C. Straub, A. Teissandier, D. Onesime, V. Loux, C. Monnet, F. Irlinger, S. Landaud, M. N. Leclercq-Perlat, P. Bento, S. Fraud, J. F. Gibrat, J. Aubert, F. Fer, E. Guedon, N. Pons, S. Kennedy, J. M. Beckerich, D. Swennen, and P. Bonnarme. 2015. Overview of a surface-ripened cheese community functioning by meta-omics analyses. PLoS One 10:e124360. https://doi.org/10 .1371/journal.pone.0124360.

Escobar-Zepeda, A., A. Sanchez-Flores, and M. Quirasco Baruch. 2016. Metagenomic analysis of a Mexican ripened cheese reveals a unique complex microbiota. Food Microbiol. 57:116-127. https:// doi.org/10.1016/j.fm.2016.02.004.

European Economic Community. 1986. Council Directive 86/609/ EEC. Accessed April 8, 2020. https://eur-lex.europa.eu/eli/dir/ 1986/609/oj.

European Union. 2010. Directive 2010/63/EU of the European Parliament and of the council. Accessed April 8, 2020. https://eur-lex .europa.eu/LexUriServ/LexUriServ.do?uri=OJ:L:2010:276:0033: 0079:EN:PDF

Falardeau, J., K. Keeney, A. Trmčić, D. Kitts, and S. Wang. 2019. Farm-to-fork profiling of bacterial communities associated with an artisan cheese production facility. Food Microbiol. 83:48-58. https: //doi.org/10.1016/j.fm.2019.04.002.

Grossiord, B., E. E. Vaughan, E. Luesink, and W. M. De Vos. 1998. Genetics of galactose utilisation via the Leloir pathway in lactic acid bacteria. Lait 78:77-84. https://doi.org/10.1051/lait:1998110.

Gunnewijk, M. G., P. T. van den Bogaard, L. M. Veenhoff, E. H. Heuberger, W. M. De Vos, M. Kleerebezem, O. P. Kuipers, and B. Poolman. 2001. Hierarchical control versus autoregulation of carbohydrate utilization in bacteria. J. Mol. Microbiol. Biotechnol. $3: 401-413$

Hamer, L., D. N. Coleman, and A. E. Relling. 2018. The effects of supplementing increasing doses of EPA and DHA fatty acids to ewes in late gestation on ewe performance and milk production and offspring performance and plasma metabolites. J. Anim. Sci. 96(suppl_2):257-258. https://doi.org/10.1093/jas/sky073.478.

Ianni, A., F. Bennato, C. Martino, D. Innosa, L. Grotta, and G. Martino. 2019a. Effects of selenium supplementation on chemical composition and aromatic profiles of cow milk and its derived cheese. J. Dairy Sci. 102:6853-6862. https://doi.org/10.3168/jds .2019-16382.

Ianni, A., D. Innosa, C. Martino, F. Bennato, and G. Martino. 2019b. Compositional characteristics and aromatic profile of caciotta cheese obtained from Friesian cows fed with a dietary supplementation of dried grape pomace. J. Dairy Sci. 102:1025-1032. https: /doi.org/10.3168/jds.2018-15590.

Ianni, A., D. Innosa, C. Martino, L. Grotta, F. Bennato, and G. Martino. 2019c. Zinc supplementation of Friesian cows: Effect on chemical-nutritional composition and aromatic profile of dairy products. J. Dairy Sci. 102:2918-2927. https://doi.org/10.3168/ jds.2018-15868.

Jung, J. Y., S. H. Lee, J. M. Kim, M. S. Park, J. W. Bae, Y. Hahn, E. L. Madsen, and C. O. Jeon. 2011. Metagenomic analysis of kimchi, a traditional Korean fermented food. Appl. Environ. Microbiol. 77:2264-2274. https://doi.org/10.1128/AEM.02157-10.

Kilcawley, K. N. 2017. Cheese flavour. Pages 443-474 in: Fundamentals of Cheese Science. P. F. Fox, T. P. Guinee, T. M. Cogan, and P. L. H. McSweeney, ed. Springer, New York, NY.

Kim, D., L. Song, F. P. Breitwieser, and S. L. Salzberg. 2016. Centrifuge: Rapid and sensitive classification of metagenomic sequences. Genome Res. 26:1721-1729.

Liu, C. L., Z. Q. Li, and X. J. Feng. 2012. Effects of daidzein or genistein on proliferation and antioxidation of mammary epithelial cell of dairy cow in vitro. Adv. Mat. Res. 343:649-654. https://doi.org/ 10.4028/www.scientific.net/AMR.343-344.649.

Liu, D. Y., S. J. He, E. H. Jin, S. Q. Liu, Y. G. Tang, S. H. Li, and L. T. Zhong. 2013. Effect of daidzein on production performance and serum antioxidative function in late lactation cows under heat stress. Livest. Sci. 152:16-20. https://doi.org/10.1016/j.livsci.2012 .12 .003 .

Luo, Z., S. Yu, Y. Zhu, J. Zhang, W. Xu, and J. Xu. 2018. Effect of various levels of isoflavone aglycone-enriched fermented soybean 
meal on redox status, serum hormones and milk quality in ewes. S. Afr. J. Anim. Sci. 48:673-682.

Martinez-Arbizu, P. 2019. PairwiseAdonis: pairwise multilevel comparison using adonis. $\mathrm{R}$ package version 2.4-5. Accessed April 6 , 2020. https://github.com/pmartinezarbizu/pairwiseAdonis

McMurdie, P. J., and S. Holmes. 2013. Phyloseq: An R package for reproducible interactive analysis and graphics of microbiome census data. PLoS One 8:e61217. https://doi.org/10.1371/journal.pone .0061217 .

McSweeney, P. L., and M. J. Sousa. 2000. Biochemical pathways for the production of flavour compound in cheeses during ripening: A review. Lait 80:293-324. https://doi.org/10.1051/lait:2000127.

Mierliță, D. 2018. Effects of diets containing hemp seeds or hemp cake on fatty acid composition and oxidative stability of sheep milk. S. Afr. J. Anim. Sci. 48:504-515. https://doi.org/10.4314/sajas.v48i3 11.

Montel, M. C., S. Buchin, A. Mallet, C. Delbes-Paus, D. A. Vuitton, N. Desmasures, and F. Berthier. 2014. Traditional cheeses: Rich and diverse microbiota with associated benefits. Int. J. Food Microbiol. 177:136-154. https://doi.org/10.1016/j.ijfoodmicro.2014 .02 .019 .

Nalbantoglu, U., A. Cakar, H. Dogan, N. Abaci, D. Ustek, K. Sayood, and H. Can. 2014. Metagenomic analysis of the microbial community in kefir grains. Food Microbiol. 41:42-51. https://doi.org/10 1016/j.fm.2014.01.014

Pastink, M. I., B. Teusink, P. Hols, S. Visser, W. M. De Vos, and J. Hugenholtz. 2009. Genome-scale model of Streptococcus thermophilus LMG18311 for metabolic comparison of lactic acid bacteria. Appl. Environ. Microbiol. 75:3627-3633. https://doi.org/10.1128/ AEM.00138-09.

Peserico, A., M. Marcacci, D. Malatesta, M. Di Domenico, A. Pratelli, I. Mangone, N. D'Alterio, F. Pizzurro, F. Cirone, G. Zaccaria, C. Cammà, and A. Lorusso. 2019. Diagnosis and characterization of canine distemper virus through sequencing by MinION nanopore technology. Sci. Rep. 9:1714. https://doi.org/10.1038/s41598-018 $-37497-4$.

Quigley, L., R. McCarthy, O. O'Sullivan, T. P. Beresford, G. F. Fitzgerald, R. P. Ross, C. Stanton, and P. D. Cotter. 2013. The microbial content of raw and pasteurized cow milk as determined by molecular approaches. J. Dairy Sci. 96:4928-4937. https://doi .org $/ 10.3168 /$ jds.2013-6688.

Salaün, F., B. Mietton, and F. Gaucheron. 2005. Buffering capacity of dairy products. Int. Dairy J. 15:95-109. https://doi.org/10.1016/ j.idairyj.2004.06.007.

Schirone, M., R. Tofalo, G. Perpetuini, A. Manetta, P. Di Gianvito, F. Tittarelli, N. Battistelli, A. Corsetti, G. Suzzi, and G. Martino. 2018. Influence of iodine feeding on microbiological and physicochemical characteristics and biogenic amines content in a raw ewes' milk cheese. Foods 7:108. https://doi.org/10.3390/foods7070108.

Smeriglio, A., E. M. Galati, M. T. Monforte, F. Lanuzza, V. D'Angelo, and C. Circosta. 2016. Polyphenolic compounds and antioxidant activity of cold-pressed seed oil from Finola cultivar of Cannabis sativa L. Phytother. Res. 30:1298-1307. https://doi.org/10.1002/ ptr.5623.

Wolfe, B. E., J. E. Button, M. Santarelli, and R. J. Dutton. 2014. Cheese rind communities provide tractable systems for in situ and in vitro studies of microbial diversity. Cell 158:422-433. https:// doi.org/10.1016/j.cell.2014.05.041.

\section{ORCIDS}

A. Ianni () https://orcid.org/0000-0003-3102-6804

M. Di Domenico (10 https://orcid.org/0000-0003-4795-8323

F. Bennato @ https://orcid.org/0000-0001-9030-4881

G. Martino @ https://orcid.org/0000-0002-7878-9318 